\title{
A New Construction of the Sierpinski Triangles with Galilean Transformations
}

\author{
Elif Aybike Büyükyılmaz*, Yusuf Yaylı and İsmail Gök \\ (Communicated by Bülent ALTUNKAYA)
}

\begin{abstract}
The aim of the paper to construct the Sierpinski triangle with deterministic algorithm iteration method in the Galilean plane $\mathbb{G}^{2}$. Self-similar figures which are the result of similarity IFS, reveal the most fundamental characteristic properties of fractals. We investigate the effects of rotation matrix $\left[\begin{array}{ll}1 & 0 \\ \varphi & 1\end{array}\right]$ to Sierpinski triangle under the iterations in Galilean plane and compare with the Euclidean plane. Furthermore, we obtain Galilean self-similarity system and Galilean box-counting dimension using the similar idea in Euclidean plane.

Keywords: Fractal; Dimension; Iteration; Galilean transformation.

AMS Subject Classification (2010): 24A80; 11K55; 39B12; $53 A 35$.

${ }^{*}$ Corresponding author
\end{abstract}

\section{Introduction}

A fractal is a fascinating mathematical set known as expanding or evolving symmetry. It is different from other geometric figures because it is a natural phenomenon. The word "fractal" was coined by Benoit Mandelbrot in 1975 and was derived from the Latin fractus meaning "broken" or "fractured". Although there are many definitions about fractals, Mandelbrot defined that they are rough or fragmented geometric shapes which are made up smaller copies of itself [17]. Two important features of fractals are self-similarity and fractal dimension. An object is precisely or nearly self-similar if it looks approximately the same on any scale. Self-similarity is an essential property of a fractal object and may be quantified by a fractal dimension. The dimension is a characteristic value which gives some geometric information about fractals. It can be any real number and this is its an unusual feature from other geometric objects behind it. The historical roots of the definition of fractal dimension were given in Haussdorff's [14] and Falconer's studies ([9], [10], [11]). There are many different definitions of fractal dimension. One of the method is the box-counting which is widely used for the reason that its simplicity ([11], [18]). Fractals are used in some applications of geology, psychology and medicine ([4], [10], [17], [19]). Mathematical introduction about fractals was obtained by Mandelbrot [17], Barnsley [2] and concepts of iterated functions and self-similarity by Edgar [8]. Many fractals can be generated by mathematical systems which a collection of transformations. These systems called iterated function system (IFS) were first studied by Hutchinson in 1981 [15] and then Barnsley and Demko [3] gave global construction of the fractals. Also there are two type of algorithms to generate fractals, called deterministic and random iteration algorithms. Deterministic iteration depends on the specific rule or recursive algorithm. In literature Sierpinski Gasket (1916), Koch Curve (1904), Cantor Set (1872) can be given as the best known deterministic fractals and details of them are given by Peitgen ([21], [22]). Sierpinski triangle, which is a perfect self-similar fractal, was named after Waclaw Sierpinski in 1916 and some studies generated the Sierpinski triangle (gasket) and carpet with computer technology [23]. In recent years, Sierpinski-type fractals and

Received : 25-January-2016, Accepted : 8-April-2016 
mathematical properties have been studied using computer programming ([5], [16]) and also have been studied their mathematical background ([18], [24], [26]). In this letter, we want to obtain some Sierpinski triangles using a new idea. First of all, we obtain them in Galilean 2-plane using the shear motion of the Galilean 2-plane.

\section{Preliminaries}

A fractal which is detailed on arbitrarily small scales, with some degree of self-similarity is an object. An affine transformation on the Euclidean plane $F: \mathbb{R}^{2} \rightarrow \mathbb{R}^{2}$ is of the form

$$
F(x, y)=(a x+b y+e, c x+d y+f)=\left(x^{\prime}, y^{\prime}\right)
$$

where $a, b, c, d, e, f \in \mathbb{R}$ and $a d-b c \neq 0$. We can also rewrite the last equation in matrix form as follows:

$$
F\left(\left[\begin{array}{l}
x \\
y
\end{array}\right]\right)=\left[\begin{array}{ll}
a & b \\
c & d
\end{array}\right]\left[\begin{array}{l}
x \\
y
\end{array}\right]+\left[\begin{array}{l}
e \\
f
\end{array}\right]
$$

The matrix $\left[\begin{array}{ll}a & b \\ c & d\end{array}\right]$ can be written in the form of $\left[\begin{array}{cc}\cos \theta & -\sin \theta \\ \sin \theta & \cos \theta\end{array}\right]$ which corresponds to a rotation $e^{i} \theta$ or $\cos \theta+i \sin \theta$ in Euclidean 2-plane.

Affine transformations which are composition of rotation, translations, dilations and shears are linear transformations. Self-affine sets are attractors of IFSs where all of the maps are contracting affine transformations on Euclidean space and have been studied recently in ([6], [12], [20]). Self-similar sets in Euclidean space are a very special class of self-affine sets. So we can define the similarity transformation.

A similarity in $\mathbb{R}^{2}$ is a function $F_{i}: \mathbb{R}^{2} \rightarrow \mathbb{R}^{2}$ satisfying

$$
|F(x)-F(y)|=r|x-y|, \quad x, y \in \mathbb{R}^{2}
$$

for some constant $r$. If $\left\{F_{1}, F_{2}, \ldots, F_{n}\right\}$ is a finite family of similarities, than it is known ([1], [10]) that there exists a unique non-empty compact set $S \subset \mathbb{R}^{2}$ such that

$$
S=\bigcup_{i=1}^{k} F_{i}(S),
$$

called the generator or invariant set of the $F_{i}$ iterated function system ([12], [15]). For $r<1$ the transformation is called contraction similarity.

Similarity transformations are the rigid motions (reflection, translation, and rotation) that preserve distance and angles. An important concept related to the similarity transformations is that of invariant. Geometrical similarity can be define by saying that two figures are similar if all the ratios between corresponding lengths, angles or areas are identical. Fractal dimension which is a measure is also useful for comparing two fractal objects. The basic principle to estimate fractal dimension is based on the concept of self-similarity. Then lets give the definition of box-counting dimension which is the aim of the study via Galilean transformations.

Definition 2.1 (Box-counting dimension). Box-counting dimension of a bounded set $A$ in Euclidean space, i.e. $D$, is defined by

$$
D=\lim _{r \rightarrow 0} \frac{\log N(r)}{\log \left(\frac{1}{r}\right)}
$$

where $N(r)$ is the smallest number of boxes (closed sets)/discs of side length/radius $r$ that cover $A$ ([11], [16]).

Then lets introduce and create Sierpinski triangle. The Sierpinski triangle, also known as the Sierpinski gasket, consists of three self-similar pieces corresponding to the three functions in the IFS. Triangle has the same basic shape of itself on any different part of it, this is its self-similarity property.

\subsection{Construction of Sierpinski triangle}

First of all we consider an equilateral triangle $S_{0}$ with side unit lengths where points $(0,0),(0.5,0.86),(1,0)$. The first iteration of $F$ gives us $S_{1}$. We can see that this is the same dividing $S_{0}$ into four smaller equilateral triangle and remove the middle triangle. The second iteration gives us $S_{2}$ which is the same as dividing each triangle in $S_{1}$ 


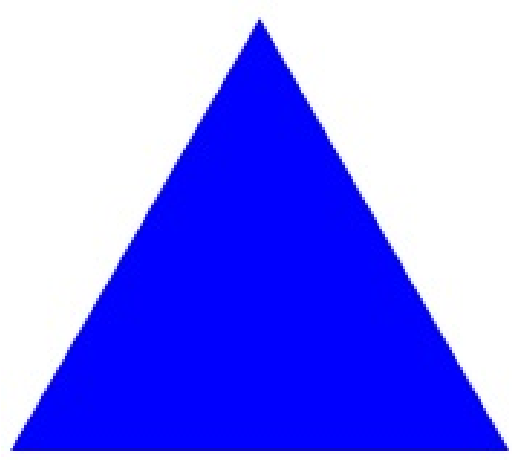

(a) $S_{0}$

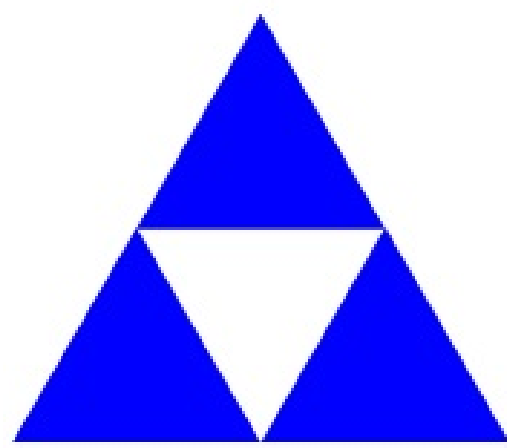

(b) $S_{1}$

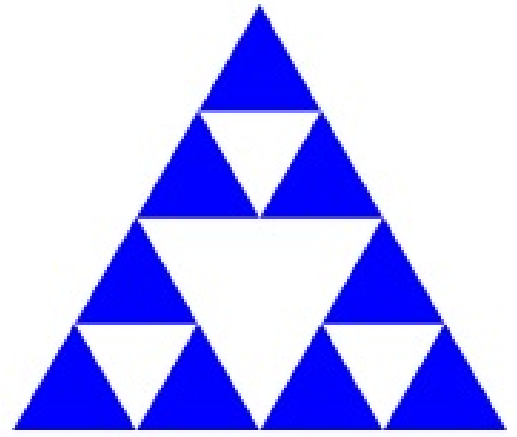

(c) $S_{2}$

Figure 1. Two iteration of Sierpinski triangle

into four smaller triangles and again removing the middle triangle from each of the larger triangles.

If we continue with this process we see that the Sierpinski triangle can be defined as $S=\bigcap_{k \in \mathbb{N}} S_{k}$, where each $S_{k}$ consists of $3^{k}$ triangles with side lengths $\frac{1}{2^{k}}$.

Then the iterated function system, $F=\left\{F_{1}, F_{2}, F_{3}\right\}$ where the $F_{i}: \mathbb{R}^{2} \rightarrow \mathbb{R}^{2}$ for equilateral Sierpinski triangle are constructed by

$$
\begin{aligned}
& F_{1}\left(\left[\begin{array}{l}
x \\
y
\end{array}\right]\right)=\left[\begin{array}{cc}
0.5 & 0 \\
0 & 0.5
\end{array}\right]\left[\begin{array}{l}
x \\
y
\end{array}\right] \\
& F_{2}\left(\left[\begin{array}{l}
x \\
y
\end{array}\right]\right)=\left[\begin{array}{cc}
0.5 & \\
0 & 0.5
\end{array}\right]\left[\begin{array}{l}
x \\
y
\end{array}\right]+\left[\begin{array}{c}
0.5 \\
0
\end{array}\right] \\
& F_{3}\left(\left[\begin{array}{l}
x \\
y
\end{array}\right]\right)=\left[\begin{array}{cc}
0.5 & \\
0 & 0.5
\end{array}\right]\left[\begin{array}{l}
x \\
y
\end{array}\right]+\left[\begin{array}{l}
0.25 \\
0.43
\end{array}\right]
\end{aligned}
$$

respectively.

The Sierpinski triangle, $S$ (Fig. 2), is the invariant set of $F$, i.e.,

$$
S=\bigcup_{i=1}^{k} F_{i}(S)=F_{1}(S) \cup F_{2}(S) \cup F_{3}(S),
$$

and has zero area.

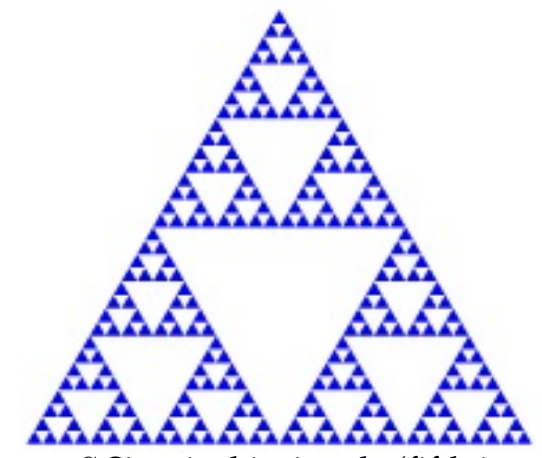

Figure 2. $S$ Sierpinski triangle (fifth iteration)

The box-counting dimension is calculated as following: 


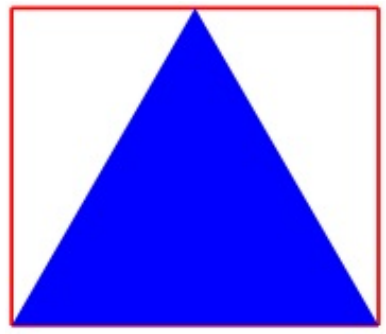

(a) $S_{0}$

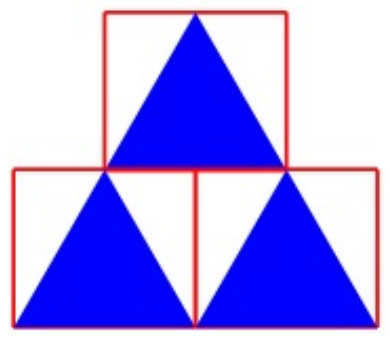

(b) $S_{1}$

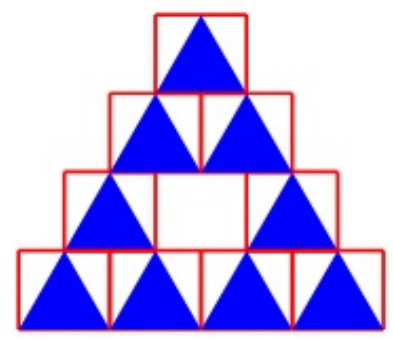

(c) $S_{2}$

$r_{0}=1, N\left(r_{0}\right)=1 \quad r_{1}=\frac{1}{2}, N\left(r_{1}\right)=3 \quad r_{2}=\frac{1}{4}, N\left(r_{2}\right)=9$

Figure 3. Box-counting dimension of Sierpinski triangle in Euclidean plane

For $S_{n}$, the scaling factor $r$ and the boxes number are $r_{n}=\left(\frac{1}{2}\right)^{n}, N\left(r_{n}\right)=3^{n}$. From (2.5) the box-counting dimension of Sierpinski triangle is $D=\frac{\log 3}{\log 2} \approx 1.585$. And from (2.1) and (2.4) we can write IFS tables for equilateral Sierpinski triangle easily:

\begin{tabular}{cccccccc}
\hline$F$ & $a$ & $b$ & $c$ & $d$ & $e$ & $f$ & $\theta$ \\
\hline 1 & 0.5 & 0 & 0.5 & 0 & 0 & 0 & 0 \\
2 & 0.5 & 0 & 0.5 & 0 & 0.5 & 0 & 0 \\
3 & 0.5 & 0 & 0.5 & 0 & 0.25 & 0.43 & 0
\end{tabular}

Table 1. IFS for Equilateral Sierpinski Triangle

If we add rotation with different $\theta$ angles to the IFS of the equilateral Sierpinski triangle we get (Fig. 4). $\theta_{L}, \theta_{R}$ and $\theta_{T}$ are the angles of the transformations $F_{1}, F_{2}$ and $F_{3}$ respectively.

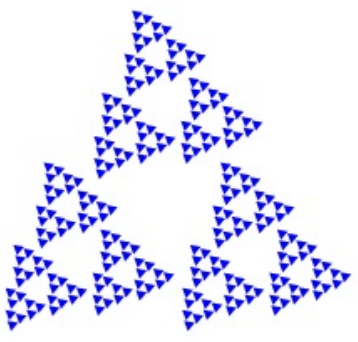

(a) $\theta_{L}=\theta_{R}=\theta_{T}=10^{\circ}$

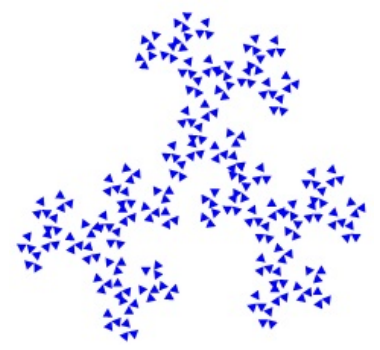

(c) $\theta_{L}=84^{\circ}, \theta_{R}=60^{\circ}$, $\theta_{T}=47^{\circ}$

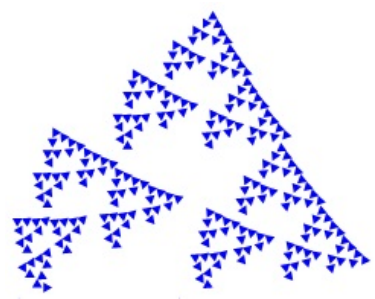

(b) $\theta_{L}=30^{\circ}, \theta_{R}=5^{\circ}$, $\theta_{T}=0^{\circ}$

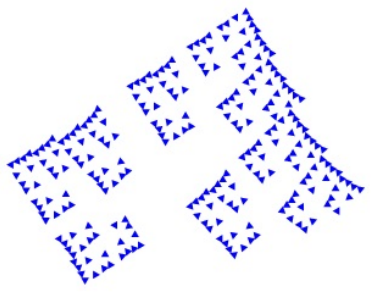

(d) $\theta_{L}=90^{\circ}, \theta_{R}=10^{\circ}$, $\theta_{T}=0^{\circ}$

Figure 4. $\theta_{L}-\theta_{R}-\theta_{T}$ rotated Sierpinski fractals

$\theta_{L}-\theta_{R}-\theta_{T}$ rotated self-similar Sierpinski fractals (Fig. 4) have the same box-counting dimension with $\theta=0$ rotated Sierpinski triangle (Fig. 1). Because all of them are created by similarity transformations which preserve geometric properties of the original shape as length, angle and area. (One of them, b) is shown below: 


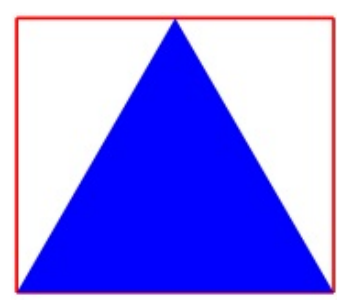

(a) $M_{0}$

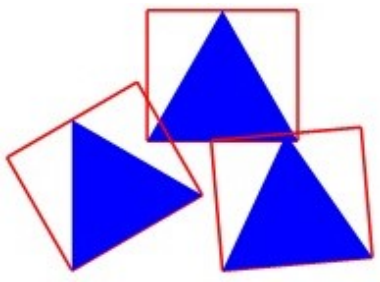

(b) $M_{1}$

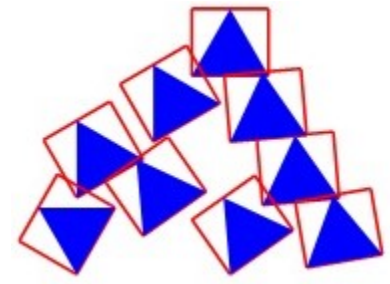

(c) $M_{2}$

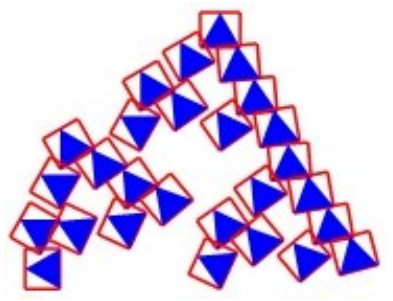

(d) $M_{3}$

$r_{0}=1, N\left(r_{0}\right)=1$

$r_{2}=\frac{1}{4}, N\left(r_{2}\right)=9$

$r_{3}=\frac{1}{8}, N\left(r_{3}\right)=27$

Figure 5. Box-counting dimension of rotating Sierpinski triangle

Each of these variations of the Sierpinski triangle is self-similar with 3 non-overlapping copies of itself. Each scaled by the factor $r=\frac{1}{2}$ and each attractor has the same box-counting dimension, $D$, as that of the Sierpinski triangle, i.e., which is calculated with $D=\frac{\log 3}{\log 2} \approx 1.585$.

\section{Galilean Plane and Galilean Transformations}

In the last two centuries new types of geometries have been invented and developed besides Euclidean geometry. These geometries are distinguished with their metric properties. Measuring lengths between two points and angles between lines relates to the metric. Galilean geometry is one of these geometries [26].

The set of dual numbers $\mathbb{D}=\left\{z=x+\varepsilon y \mid x, y \in \mathbb{R}, \varepsilon \neq 0, \varepsilon^{2}=0\right\}$ is a commutative ring. $\mathbb{R}^{2}$ with inner product given by

$$
\begin{aligned}
\langle,\rangle_{g}: \mathbb{D} \times \mathbb{D} & \longrightarrow \mathbb{R} \\
\left(z_{1}, z_{2}\right) & \longrightarrow\left\langle z_{1}, z_{2}\right\rangle_{g}=x_{1} x_{2}, \quad x_{1} \neq x_{2} .
\end{aligned}
$$

where $z_{1}=x_{1}+\varepsilon y_{1}, z_{2}=x_{2}+\varepsilon y_{2} \in \mathbb{D}$ is the Galilean plane and shown $\left(\mathbb{R}^{2},\langle,\rangle_{g}\right)$ or $\mathbb{G}^{2}$.

On the Galilean Plane, the distance $d(A, B)$ between two points $A\left(x_{1}, y_{1}\right)$ and $A\left(x_{2}, y_{2}\right)$ is defined by the formula

$$
d_{A B}=d(A, B)=\left|x_{2}-x_{1}\right|
$$

and this length $\left(P P_{1}\right)$ is the projection of the $A B$ on the x-axis (Fig. 6a). If the points $A\left(x_{1}, y_{1}\right)$ and $B\left(x_{2}, y_{2}\right)$ belong to the same special line, i.e., $x_{1}=x_{2}$ (Fig. $6 \mathrm{~b}$ ), then $d(A, B)$ is a special distance and defined by following formula, [26],

$$
\delta(A, B)=\left|y_{2}-y_{1}\right| .
$$

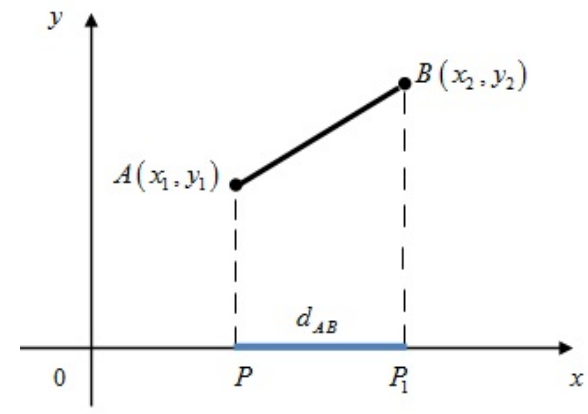

(a) $d_{A B}$ distance in $\mathbb{G}^{2}$

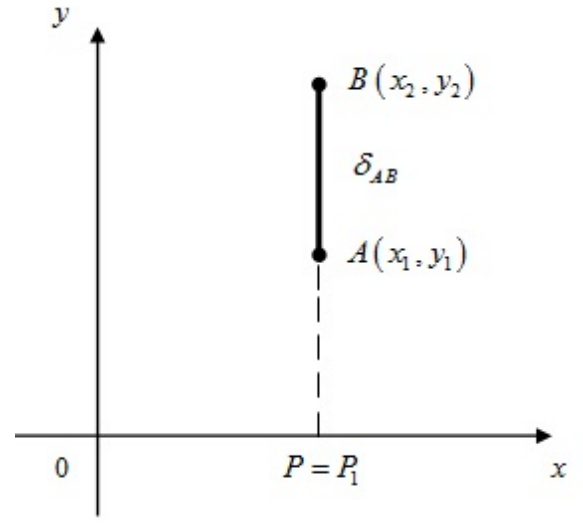

(b) $\delta_{A B}$ special distance in $\mathbb{G}^{2}$

Figure 6 
Then, the Galilean metric $d$ is defined by

$$
d(A, B)= \begin{cases}\left|x_{1}-x_{2}\right|, & x_{1} \neq x_{2} \\ \left|y_{1}-y_{2}\right|, & x_{1}=x_{2}\end{cases}
$$

The modulus of a point $X=(x, y)$ in Galilean plane is also defined by

$$
\|X\|= \begin{cases}|x|, & x \neq 0 \\ |y|, & x=0\end{cases}
$$

A circle is the set of points a given distance from the center. The set of all points on the Galilean plane that satisfy the equation $\|X\|_{d}=|x|=r>0$ is the two special lines $x= \pm r$ and is called the Galilean circle (Fig. 7a) [26].

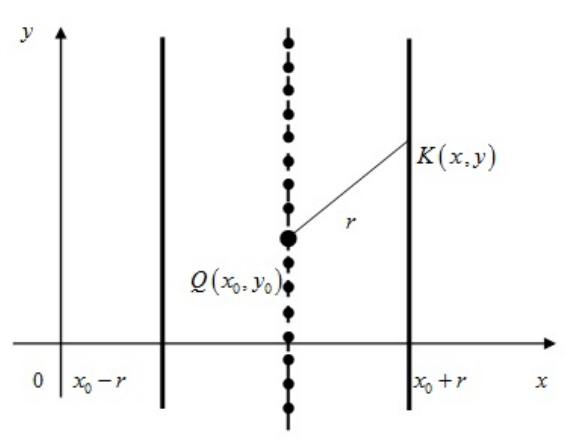

(a) $K(x, y)$ Galilean circle

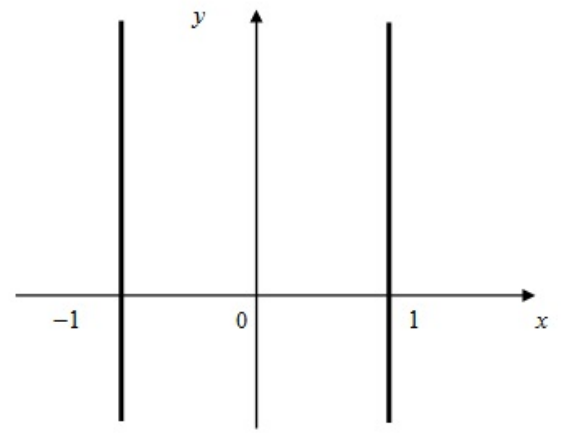

(b) $S_{G}^{1}$ Galilean unit circle

Figure 7

It is clear that for $r=1$ the Galilean unit circle (Fig. 7b) has two branches, the vertical lines $x= \pm 1$, and is defined by

$$
S_{G}^{1}=\{P(x, y) \mid x= \pm 1, y \in \mathbb{R}\}
$$

$A B C$ is any triangle in Galilean plane, (Fig. 10) there are following equations between $d_{B C}=a, d_{A C}=b$, $d_{A B}=c$ side lengths and $\delta_{b c}=\widehat{A}, \delta_{c a}=\widehat{B}, \delta_{a b}=\widehat{C}$ angles, [26]:

$$
\begin{aligned}
d_{B C}+d_{A C} & =d_{A B} \\
\widehat{A}+\widehat{B} & =\widehat{C}
\end{aligned}
$$

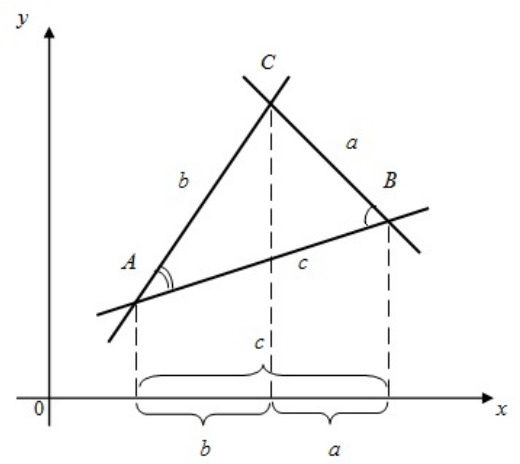

Figure 8. $A B C$ triangle in Galilean plane

The angle between two ordinary lines $y=m_{1} x+n_{1}$ and $y=m_{2} x+n_{2}$ intersecting at a point $Q\left(x_{0}, y_{0}\right)$ (Fig. 9) is defined by

$$
\delta_{l_{1} l_{2}}=\left|m_{2}-m_{1}\right|
$$




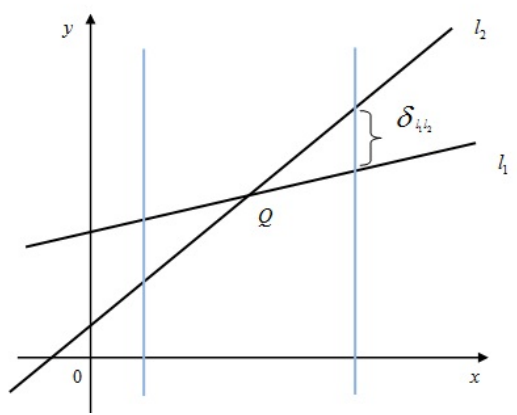

Figure 9. Angle between two lines in Galilean plane

The Galilean $\operatorname{Cosine} \operatorname{cosg}(\varphi)$ and the Galilean Sine $\operatorname{sing}(\varphi)$ can be defined by the following equations:

$$
x=\operatorname{rcosg}(\varphi), \quad y=r \operatorname{sing}(\varphi)
$$

where $z$ is a point on the Galilean unit circle with centered at $O$ and for all $\varphi, \cos g(\varphi)=1$ and $\operatorname{sing}(\varphi)=\varphi$. Then the polar form of $z$ can be written

$$
z=r(\operatorname{cosg}(\varphi)+\varepsilon \operatorname{sing}(\varphi))
$$

Such the analogue of Euler's formula for the dual numbers, every dual number $z=x+\varepsilon y$ with $r$ modulus can be writtten

$$
z=r e^{\varepsilon \varphi}=r(1+\varepsilon \varphi)=r(\cos g(\varphi)+\varepsilon \operatorname{sing}(\varphi))
$$

The equation $1+\varepsilon \varphi=e^{\varepsilon \varphi}$ can be derived from a power series expansion $e^{x}$ for all $\varphi$ and $r=1$, [1]. A rotation in Galilean plane by $e^{\varepsilon \varphi}$ corresponds to multiplication by the matrix, $\left[\begin{array}{ll}1 & 0 \\ \varphi & 1\end{array}\right]$ and also is called Shear transformation. Thus, the dual number multiplication can be used to produce a shear transformation [26].

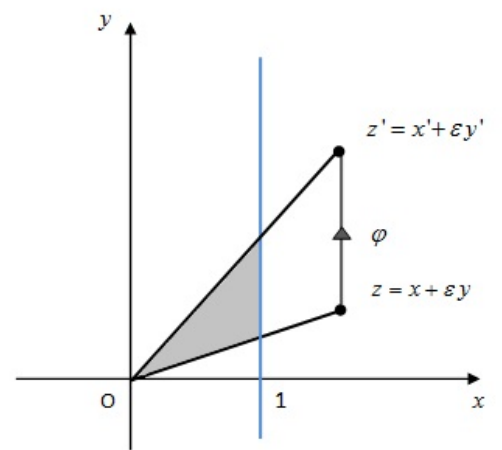

Figure 10. Rotation in Galilean plane

Shear transformation is the most interesting application of dual numbers. Furthermore, they are also used in spatial geometry and in non-Euclidean geometry [26]. In Galilean plane unit dual numbers play the same role with the unit complex numbers in Euclidean plane. Thus the rotation on Galilean plane can be defined by in matrix form

$$
\begin{gathered}
{\left[\begin{array}{l}
x^{\prime} \\
y^{\prime}
\end{array}\right]=\left[\begin{array}{ll}
1 & 0 \\
\varphi & 1
\end{array}\right]\left[\begin{array}{l}
x \\
y
\end{array}\right]} \\
\left\{\begin{array}{l}
x^{\prime}=x \\
y^{\prime}=\varphi x+y
\end{array}\right.
\end{gathered}
$$

Galilean transformation given by the formula

$$
\left\{\begin{array}{l}
x^{\prime}=x+e \\
y^{\prime}=\varphi x+y+f
\end{array} .\right.
$$


is a composition of shear and translation. Galilean transformation preserve the distance between the points where $\varphi$ shows the measure of the angle.

In Galilean geometry transformations (3.14) map

1. Lines onto lines;

2. Parallel lines onto parallel lines;

3. Collinear segments onto collinear segments;

4. A figure $F$ onto a figure $F^{\prime}$ of the same area [26].

\section{Sierpinski-type Fractals in Galilean Plane}

In this section we will introduce fractals on Galilean plane geometrically and we calculate the box-counting dimension of Galilean meaning. There are two important features of fractals which are self-similarity and fractal dimension as the same to Euclidean plane. IFSs of affine transformations in Galilean plane which have no rotation, behave the same in Euclidean plane.

As an illustration we get a triangle $L_{0}$ which has corner points $(0,0),(0.5,0.86),(1,0) . L_{0}$ under IFS (Table 1$)$ has the same shape in Euclidean and Galilean plane. Even though $L_{0}$ is an equilateral triangle in Euclidean plane, it is an isosceles triangle in Galilean plane which two side lengths are 0.5 and the other is 1 (Fig. 1). The first iteration of $F$ gives us $L_{1}$. We can see that this is the same dividing $L_{0}$ into three smaller isosceles triangle. The second iteration gives us $L_{2}$, is the same as dividing each triangle in $L_{1}$ into three smaller triangles. If we continue with this process we see that the Sierpinski triangle can be written as $L=\bigcap_{k \in \mathbb{N}} L_{k}$, where each $L_{k}$ consists of $3^{k}$ triangles with side lengths $\frac{1}{2^{k}}$.

Lets explain the rotation in Galilean plane geometrically without scaling. Let $A O B$ be the isosceles triangle (Fig. 11a) in Galilean plane which side lengths are $0.5,0.5,1$ and $\left[\begin{array}{ll}1 & 0 \\ \varphi & 1\end{array}\right]$ be the rotation matrix. If we rotate $A O B$ for $\varphi=1$ with $\left[\begin{array}{ll}1 & 0 \\ 1 & 1\end{array}\right]$ matrix, we get $A^{\prime} O B^{\prime}$ (Fig. 11b). And if we rotate $A^{\prime} O B^{\prime}$ again with $\left[\begin{array}{ll}1 & 0 \\ 1 & 1\end{array}\right]$ matrix, we get $A^{\prime \prime} O B^{\prime \prime}$ (Fig. 11c). As seen in Fig. 11, it can be said that in the Galilean plane from (3.1) $|A O|=\left|A^{\prime} O\right|=\left|A^{\prime \prime} O\right|=0.5$,

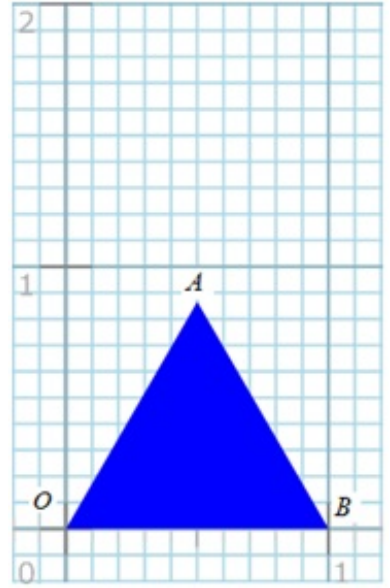

(a)

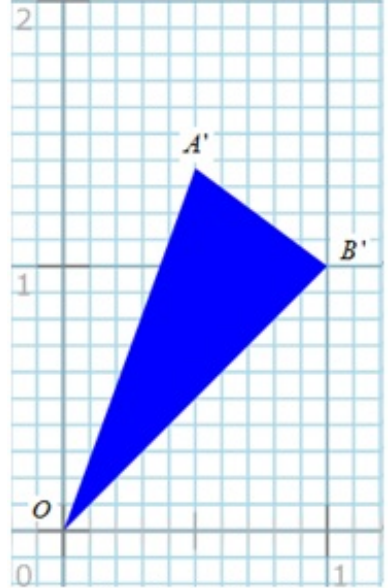

(b)

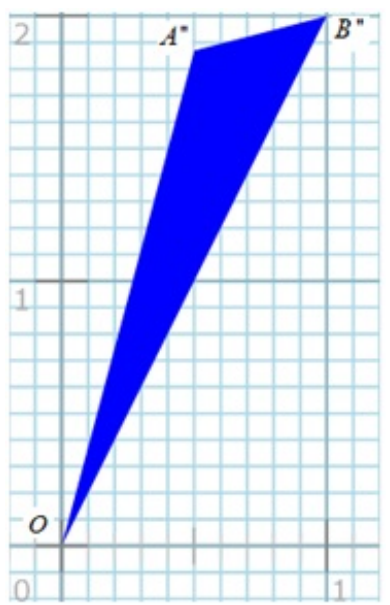

(c)

Figure 11. Rotation of $A O B$ in Galilean plane

$|A B|=\left|A^{\prime} B^{\prime}\right|=\left|A^{\prime \prime} B^{\prime \prime}\right|=0.5,|O B=| O B^{\prime}|=| O B^{\prime \prime} \mid=1$ and the angles are protected from the equation (3.7). In this case, under the $\left[\begin{array}{ll}1 & 0 \\ 1 & 1\end{array}\right]$ rotation $A O B, A^{\prime} O B^{\prime}$ and $A^{\prime \prime} O B^{\prime \prime}$ are self-similarity in Galilean meaning.

In this case, in the Galilean plane, any $A B C$ which has the vertices $A\left(x_{1}, y_{1}\right), B\left(x_{2}, y_{2}\right), C\left(x_{3}, y_{3}\right)$, rotates with the matrix $\left[\begin{array}{ll}1 & 0 \\ \varphi & 1\end{array}\right]$ and forms the new triangle of vertices $A^{\prime}\left(x_{1}^{\prime}, y_{1}^{\prime}\right), B^{\prime}\left(x_{2}^{\prime}, y_{2}^{\prime}\right)$ and $C^{\prime}\left(x_{3}^{\prime}, y_{3}^{\prime}\right)$. After the $\varphi$-shear 
transformation, this correlation can be seen:

$$
\begin{aligned}
& x_{1}^{\prime}=x_{1} \\
& x_{2}^{\prime}=x_{2} \quad x_{3}^{\prime}=x_{3} \\
& y_{1}^{\prime}=\varphi x_{1}+y_{1} \\
& y_{2}^{\prime}=\varphi x_{2}+y_{2} \\
& y_{3}^{\prime}=\varphi x_{3}+y_{3} .
\end{aligned}
$$

$A B C$ and $A^{\prime} B^{\prime} C^{\prime}$ are self-similar in Galilean meaning under the $\varphi$-shear transformation which preserves the distance and angles. There will be this general correlation between the triangles and vertices when $A B C$ rotates $\mathrm{n}$. times:

$$
\begin{aligned}
& x_{n}^{\prime}=x_{1}, \\
& y_{n}^{\prime}=n \varphi x_{1}+y_{1} .
\end{aligned}
$$

The following difference should be put forward: the self-similarity of a triangle after rotation is different from the similarity of triangles in the Galilean plane.

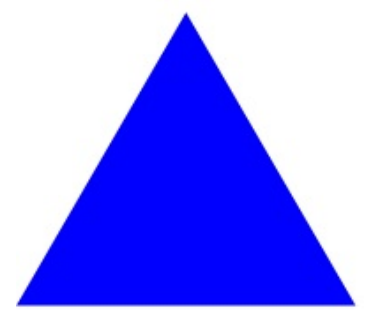

(a) $L_{0}$

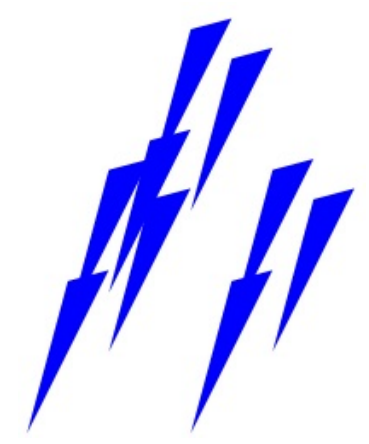

(c) $L_{2}$

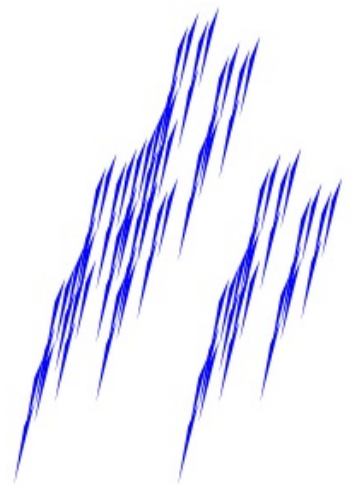

(e) $L_{4}$

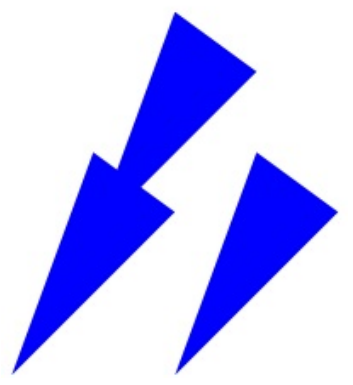

(b) $L_{1}$

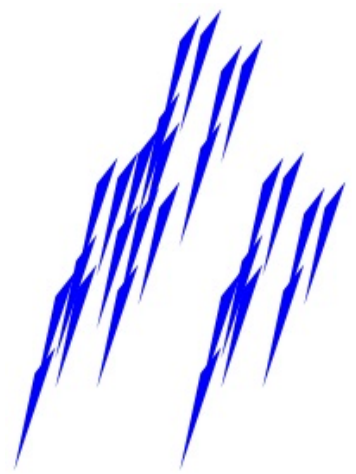

(d) $L_{3}$

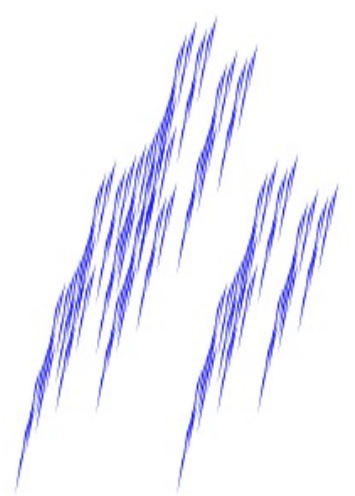

(f) $L_{5}$

Figure 12. $\varphi=1$ rotated Sierpinski triangle in Galilean plane 
Then, lets apply a Galilean rotation to IFS (Table 1) and see the result. The iterated function system, $F=$ $\left\{F_{1}, F_{2}, F_{3}\right\}$ where the $F_{i}: \mathbb{G}^{2} \longrightarrow \mathbb{G}^{2}$ for isosceles Sierpinski triangle $L_{0}$ are defined by

$$
\begin{aligned}
& F_{1}\left(\left[\begin{array}{l}
x \\
y
\end{array}\right]\right)=\left[\begin{array}{cc}
0.5 & 0 \\
0 & 0.5
\end{array}\right]\left[\begin{array}{l}
x \\
y
\end{array}\right] \\
& F_{2}\left(\left[\begin{array}{l}
x \\
y
\end{array}\right]\right)=\left[\begin{array}{cc}
0.5 & \\
0 & 0.5
\end{array}\right]\left[\begin{array}{l}
x \\
y
\end{array}\right]+\left[\begin{array}{c}
0.5 \\
0
\end{array}\right] \\
& F_{3}\left(\left[\begin{array}{l}
x \\
y
\end{array}\right]\right)=\left[\begin{array}{cc}
0.5 & \\
0 & 0.5
\end{array}\right]\left[\begin{array}{l}
x \\
y
\end{array}\right]+\left[\begin{array}{l}
0.25 \\
0.43
\end{array}\right]
\end{aligned}
$$

respectively. Hence, we can write the IFS table for $L_{0}$ easily:

\begin{tabular}{cccccccc}
\hline$F$ & $a$ & $b$ & $c$ & $d$ & $e$ & $f$ & $\theta$ \\
\hline 1 & 0.5 & 0 & 0.5 & 0.5 & 0 & 0 & 0 \\
2 & 0.5 & 0 & 0.5 & 0.5 & 0.5 & 0 & 0 \\
3 & 0.5 & 0 & 0.5 & 0.5 & 0.25 & 0.435 & 0
\end{tabular}

Table 2. IFS codes for rotating Sierpinski in Galilean plane

and the $\varphi=1$ rotated Sierpinski triangle is shown in (Fig. 12).

Then, we can define the Galilean meaning self-similarity. A similarity transformation in $\mathbb{G}^{2}$ is a function $F_{i}: \mathbb{G}^{2} \longrightarrow \mathbb{G}^{2}$ satisfying

$$
|F(x)-F(y)|=r|x-y|, \quad x, y \in \mathbb{R}^{2}
$$

for some constant $r$ which preserve the distance, angle and area measures.

When two triangles are similar in Galilean meaning, corresponding angles are congruent and corresponding sides are proportional. Finally the definition of box-counting dimension in Galilean plane is the same one in Euclidean plane. If the IFS has a Galilean rotation then we develop new approach to calculate box-counting dimension. Lets introduce this with geometrically and $L_{0}$ be the isosceles triangle. To cover $L_{0}$ we use a box which is proportioned by side and height lengths. After the first iteration the shape will be (Fig. 13b) and we cover each part of it with rotationed box which is called parallelogram.

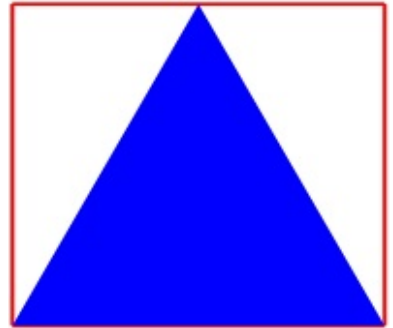

(a)

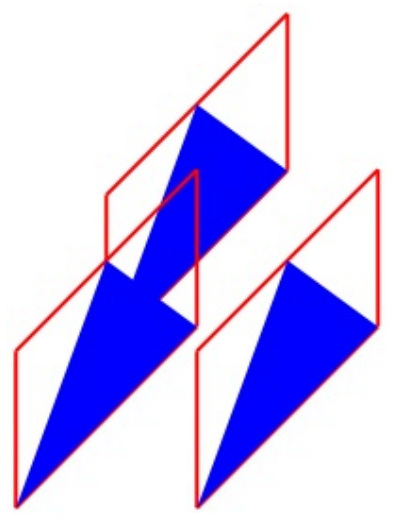

(b)

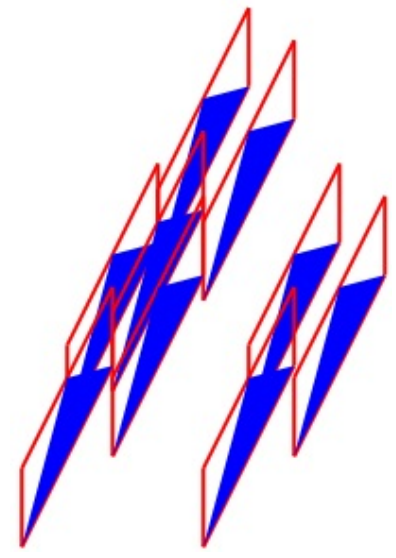

(c)

Figure 13. Box-counting with parallelogram

Under each iteration, parallelograms will be scaled, rotated and translated. So the Galilean box-counting dimension $D_{G}$ in Galilean plane is defined by

$$
D_{G}=\lim _{r \rightarrow 0}=\frac{\log N(r)}{\log \left(\frac{1}{r}\right)}
$$

where $N(r)$ is the smallest number of parallelograms of side length $r$ that cover $A$.

Examples of Galilean Sierpinski fractals formed by the similarity transformations corresponding to the $\varphi$-shear 
values of each $F_{i}$ isoscele triangle $L_{0}$ in the Galilean plane and the IFS of each fractals are given in the table below. $b$ and $c$ values in the $F\left(\left[\begin{array}{l}x \\ y\end{array}\right]\right)=\left[\begin{array}{ll}a & b \\ c & d\end{array}\right]\left[\begin{array}{l}x \\ y\end{array}\right]+\left[\begin{array}{l}e \\ f\end{array}\right]$ transformation which refer $x$-shear and $y$-shear respectively are associated with only rotational movement. Rotation and its quantity does not change fractal's box-counting Galilean dimension as the same to Euclidean plane. Thus the fractals in Fig. 14 have the same box-counting Galilean dimension which is calculated as before $D=\frac{\log 3}{\log 2} \approx 1.585$.
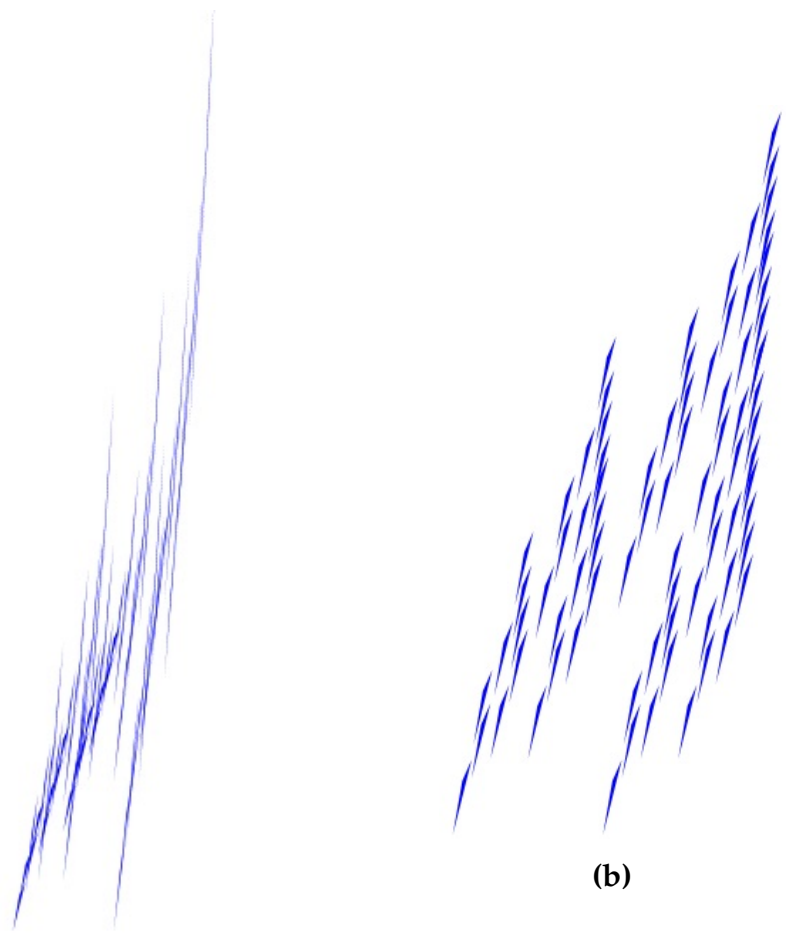

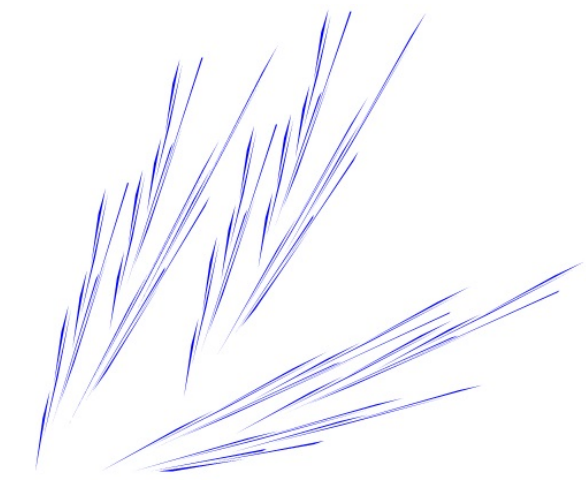

(c)

(a)
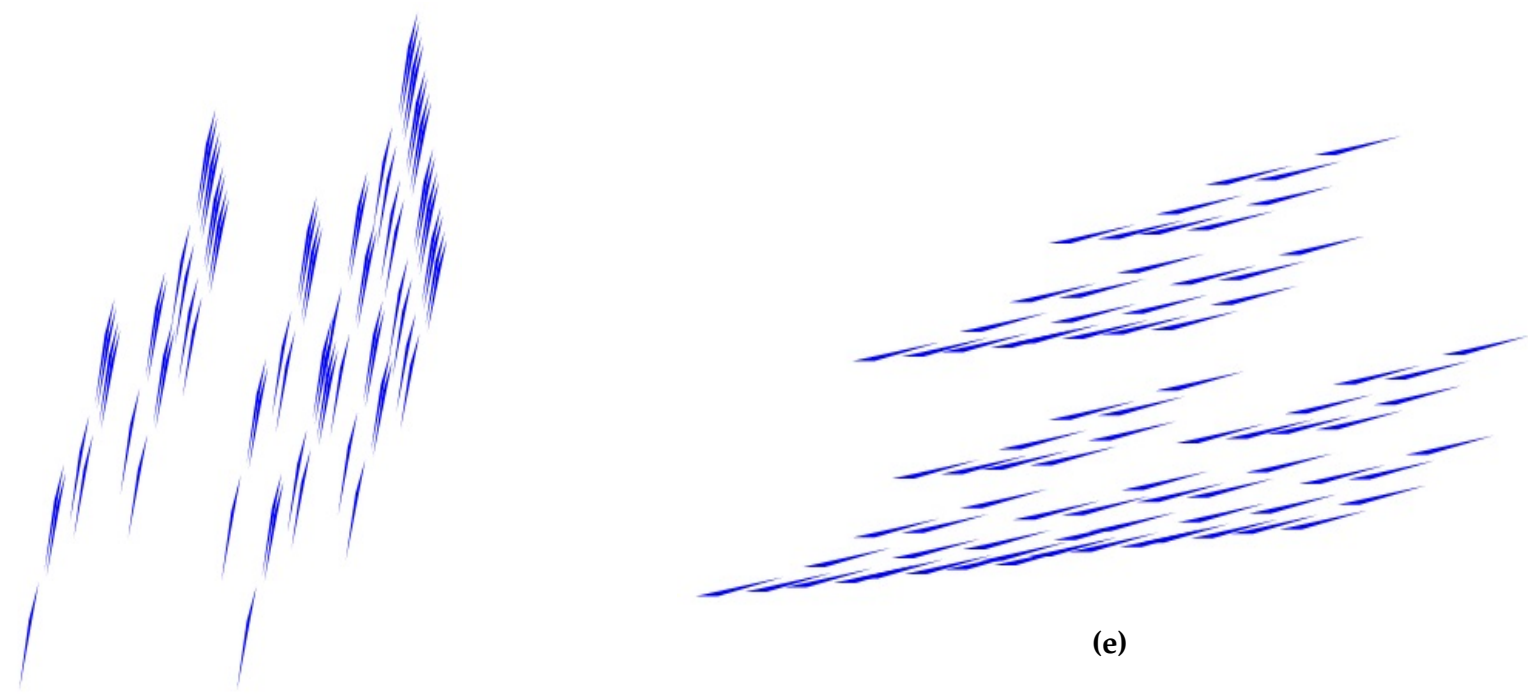

(e)

(d)

Figure 14. Sierpinski-type fractals for different $\varphi$ values in Galilean plane 
(a)

\begin{tabular}{cccccccc}
\hline$F$ & $a$ & $b$ & $c$ & $d$ & $e$ & $f$ & $\theta$ \\
\hline 1 & 1 & 0 & 1 & 1 & 0 & 0 & 0 \\
2 & 1 & 0 & 5 & 1 & 0.5 & 0 & 0 \\
3 & 1 & 0 & 1 & 1 & 0.25 & 0.5 & 0
\end{tabular}

(b)

\begin{tabular}{cccccccc}
\hline$F$ & $a$ & $b$ & $c$ & $d$ & $e$ & $f$ & $\theta$ \\
\hline 1 & 1 & 0 & 1 & 1 & 0 & 0 & 0 \\
2 & 1 & 0 & 1 & 1 & 0.5 & 0 & 0 \\
3 & 1 & 0 & 1 & 1 & 0.5 & 0.75 & 0
\end{tabular}

(c)

\begin{tabular}{cccccccc}
\hline$F$ & $a$ & $b$ & $c$ & $d$ & $e$ & $f$ & $\theta$ \\
\hline 1 & 1 & 0 & 1.4 & 1 & 0 & 0 & 0 \\
2 & 1 & 1.62 & 0 & 1 & 0.28 & 0 & 0 \\
3 & 1 & 0 & 1.23 & 1 & 0.64 & 0.32 & 0
\end{tabular}

(d)

\begin{tabular}{cccccccc}
\hline$F$ & $a$ & $b$ & $c$ & $d$ & $e$ & $f$ & $\theta$ \\
\hline 1 & 1 & 0 & 1.4 & 1 & 0 & 0 & 0 \\
2 & 1 & 0 & 1.2 & 1 & 0.7 & 0 & 0 \\
3 & 1 & 0 & 1.34 & 1 & 0.65 & 0.35 & 0 \\
\hline$F$ & $a$ & $b$ & $c$ & $d$ & $e$ & $f$ & $\theta$ \\
\hline 1 & 1 & 1 & 0 & 1 & 0 & 0 & 0 \\
\hline 2 & 1 & 1 & 0 & 1 & 0.8 & 0.1 & 0 \\
3 & 1 & 1 & 0 & 1 & 0.44 & 0.66 & 0
\end{tabular}

Table 3. IFS codes of different Sierpinski-type fractals

\section{References}

[1] Akar, M., Yüce, S. and Kuruoglu, N., One-Parameter-Planar Motion in the Galilean Plane, International Electronic Journal of Geometry, Volume 6 (2003), no.1, 79-88.

[2] Barnsley, M.F., Fractals Everywhere, 2nd ed., Academic Press, San Diego, 1993.

[3] Barnsley, M.F., and Demko, S., "Iterated function systems and the global construction of fractals", Proc. R. Soc. London, A 399 (1985), p. 243-275.

[4] Barnsley M. F., Devaney R. L., Benoit B. M., Pietgen H. O., Saupe D., Voss R. F., (1988), “The Science of Fractal Images", Springer-Verlag, USA, pp. 1-20.

[5] Barnsley M.F., et al, The science of fractal images,Springer-Verlag, New York, (1988).

[6] Bedford T., The box dimension of self-affine graphs and repellers. Nonlinearity 1 (1989), 53-71.

[7] Bedford T. and Urbanski M., The box and Hausdorff dimension of self-affine sets. Ergodic Theory Dynamical Systems 10 (1990), 627-644.

[8] Edgar G.A., Measure, Topology, and Fractal Geometry, Undergraduate Texts in Mathematics, Springer-Verlag, (1990).

[9] Falconer K J, The Geometry of Fractal Sets, Cambridge University Press (1985).

[10] Falconer K J, Fractal Geometry, Wiley, (1990).

[11] Falconer K. J., Fractal Geometry-Mathematical Foundations and Applications (John Wiley, 2nd ed. (2003).

[12] Falconer K.J., The Haussdorf dimension of self-affine fractals, Math. Proc. Cambr. Phil. Soc. 103(1988), 339-350.

[13] Falconer K.J. and Miao J., Dimensions of self-affine fractals and multifractals generated by upper-triangular matrices, Fractals 15(2007), 289.

[14] Hausdorff, Felix (1918), "Dimension und äusseres Mass", Mathematische Annalen 79 (1-2): 157-179. 
[15] Hutchinson J.E., Fractals and self-similarity, Indiana.Univ. Math. J. 30, 1981, pp. 713-749.

[16] Lu. N., Fractal imaging, Morgan Kaufmann Publishers, (1997).

[17] Mandelbrot, B., The Fractal Geometry of Nature, (1982).

[18] Mandelbrot B. B., (1984), "The Fractal Geometry of Nature", The American Mathematical Monthly, vol. 91, no. 9, pp. 594-598.

[19] Mandelbrot B. B., (1989), "Fractal Geometry: What Is It , and What Does It Do?", Proceedings of the Royal Society of London, vol. 423, pp. 2-16.

[20] McMullen C., The Hausdorff dimension of general Sierpinski carpets, Nagoya Math. J. 96,(1984), pp. 1-9.

[21] Peitgen H-O, Jürgens H and Saupe D 1992 a Chaos and Fractals: New Frontiers of Science (New York: Springer).

[22] Peitgen H-O, Jürgens H, Saupe D, Maletsky E M, Perciante T H and Yunker L E 1991 Fractals for the Classroom:Strategic Activities Volume One (New York: Springer).

[23] Reiter C.A., Sierpinski fractals and GCD's Comput. Graph. 18 885-91, (1994).

[24] Rooney J., On the three types of complex number and planar transformations, Cranfield Institute of Technology, (1978).

[25] Taylor T.D., Connectivity properties of Sierpinski relatives, Fractals. 19(4), (2011), pp. 481-506.

[26] Yaglom I.M. A simple non-Eucledian geometry and its physical basis: an elementary account of Galilean geometry and the Galilean principle of relativity. New-York: Springer-Verlag, (1979).

[27] Zhou Z.L., Hausdorff measure of Sierpinski triangle, Sci. China. Ser. A. 40(10), (1997),pp. 1016-1021.

\section{Affiliations}

\section{ELIF AYBIKE BÜYÜKYILMAZ}

AdDress: Ankara University, Dept. of Mathematics, 06100, Ankara-TURKEY.

E-MAIL: eabuyukyilmaz@ankara.edu.tr

YUSUF YAYLI

AdDRESS: Ankara University, Dept. of Mathematics, 06100, Ankara-TURKEY.

E-MAIL: yayli@science.ankara.edu.tr

İsMAIL GÖK

AdDRESS: Ankara University, Dept. of Mathematics, 06100, Ankara-TURKEY.

E-MAIL: igok@science.ankara.edu.tr 